\title{
Efficient Future Mobility with Intelligent Drivetrain Systems for Hybrids and EVs
}

\author{
Matthias Zink ${ }^{1)}$ \\ 1) Schaeffler AP RHQ \\ No. 100 Yutong Road, 200070 Shanhai, China
}

Received on June 26, 2012

\begin{abstract}
Using the motto "efficient future mobility",are supporting and shaping the path towards electric mobility with close customer collaboration.These activities are primarily focused on consistently optimizing the internal combustion drivetrain,on hybrid modules and on components and modules for E-Mobility.
\end{abstract}

KEY WORDS: EV and HV system, motor drive system, electric power conversion, heat engine, power transmission, EV and HV systems, vehicle dynamics [A2]

\section{INTRODUCTION}

The factors pushing e-mobility forward can now be clearly summarized as follows:

- Reducing $\mathrm{CO}_{2}$ emissions

- Availability of resources

- Political and legal restrictions

- Urbanization of mobility and/or local emission levels

- The trend towards a greener society

- Attaining and/or maintaining technological leadership

In addition to all social and technical reasoning, e-mobility must also provide economically viable solutions for end users. Further development of drivetrains is therefore one of the issues currently being pursued with maximum priority in the automotive industry.

In a race against e-mobility, the automotive industry is currently consistently optimizing both the engines and transmissions of internal combustion engines. The efficiency, number of gears and ratios of increasingly automatic transmissions are being further developed, in order to ensure internal combustion engines can be operated in the most fuelefficient operating points. Vehicles are gradually also being equipped with start-stop systems.

\section{HYBRIDS}

A large variety of hybrid drivetrains are currently available on the market. The "P2 Parallel Hybrid", used in the Nissan Fuga and Porsche Cayenne, is an interesting variant from a technical point of view. A hybrid module, comprising an electric motor, damper, separating clutch and actuator system, is fitted in a conventional drivetrain and facilitates the start-stop function, disconnects the internal combustion engine during coasting, is responsible for boosting, and electric driving with peak output of up to $35 \mathrm{~kW}$. The requirements placed on the hybrid module due to these functions are as follows:
- Very low mass moment of the clutch components

- High dynamics when engaging the clutch

- Good controllability in torque ranges between 50-150 Nm

- Low drag torque when clutch is open

- Facilitates tow starts up to $3000 \mathrm{rpm}$

- "Normally closed" clutch system (fail safe)

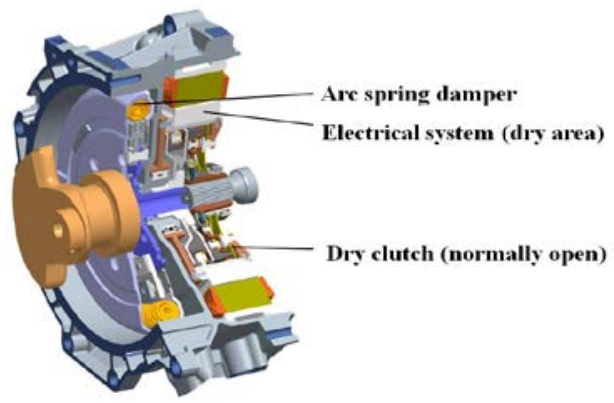

Fig.1 1st generation P2 Parallel hybrid

In addition to increased electric power, further reducing the design space required by the entire system is one of the most important requirements for the development of the next generation of P2 Parallel hybrids. To resolve this conflict of objectives, the separating clutch is integrated in the rotor and the function is split between the one-way clutch (traction direction) and conventional clutch (thrust direction).

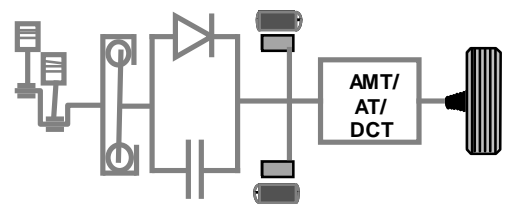

Fig.2 2nd generation P2 Parallel hybrid 


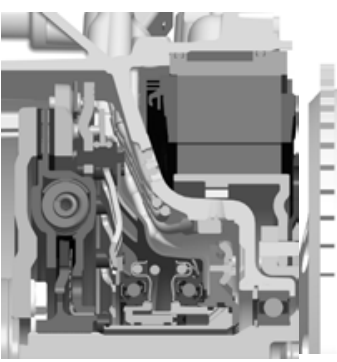

Fig.3a 1st generation ${ }^{(1)}$

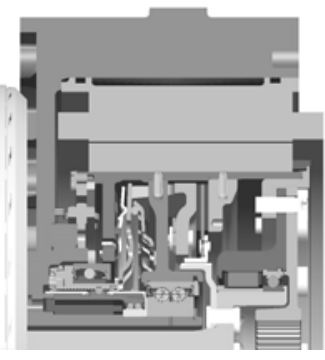

Fig.3b 2nd generation ${ }^{(1)}$ (integrated)
This innovative variant of the hybrid module with integrated clutch facilitates engine torque up to $800 \mathrm{Nm}$ (oneway clutch) and coast torque up to $150 \mathrm{Nm}$ (separating clutch). The installed electric output is $60 \mathrm{~kW}$ (continuous) and $90 \mathrm{~kW}$ (peak) (1st generation: $35 \mathrm{~kW}$ ), which provides a significantly greater range for all-electric driving ${ }^{(1)}$.

In addition, a variant of the double clutch transmission, in which one sub-transmission can be controlled by an electric motor, features very interesting technology and is expected to go into volume production in future. The advantage in this case is that the basic architecture of the transmission (hydrostatic or electromechanical actuator system and 2 sub-transmissions) in itself already offers all options for intelligent hybridization.

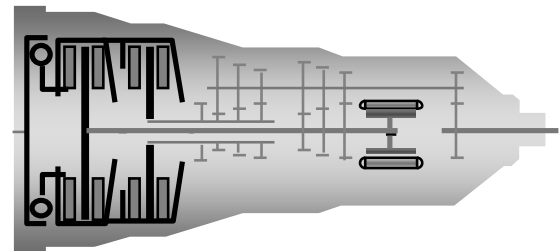

Fig.4 DCT hybrid structure

\section{INTERNAL COMBUSTION ENGINES VERSUS E- MOBILITY}

Before the technical solutions for purely electric drives favored by us are analyzed, a comparison must be made, using a vehicle from the lower mid-class as an example. The objective of this comparison is to determine from which point the power provision efficiency of fuel and of electricity actually make a vehicle powered by an electric motor feasible in terms of efficiency and $\mathrm{CO}_{2}$ emissions. The first step involves comparing an internal combustion engine (0.7 liter, 3-cylinder turbo, 70 $\mathrm{kW})$ with a permanently-excited electric motor. The internal combustion engine drivetrain is equipped with a 5-speed manual transmission and the electric motor is equipped with a single-

speed gearbox with 7:1 gear ratio. The losses from the transmissions are comparable.

3.1. Comparison of efficiency of internal combustion engines and electric vehicles

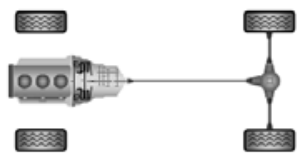

Engine: 3-cylinder turbo gasoline

Transmission: 5-speed MT
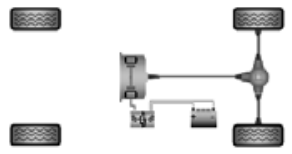

E-motor: PSM

Transmission: 1-speed reduction gear

Fig.5 comparative drivetrain structure

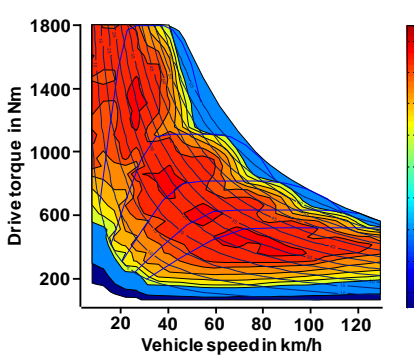

3-cylinder engine

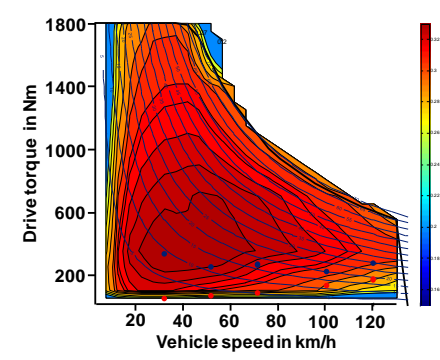

E-motor
Fig.6 efficiency comparison e-motor vs. 3 cylinder engine

The following assumptions are used as the basic assumptions for the efficiency characteristic diagram of the (permanentlyexcited) electric motor:

- Power provision efficiency 38\%, (corresponds to structure of power plants in Germany in 2008)

- Constant efficiency when charging

- Discharge efficiency corresponds with the power level

The superposition of the data maps shows that the benefit in terms of efficiency for the electric vehicle is rather low at $2-5 \%$ over a wide area of the data map. Excerpts from the NEDC (constant and acceleration proportion) have been included as an example, and even here, the advantage of the electric drive is limited.

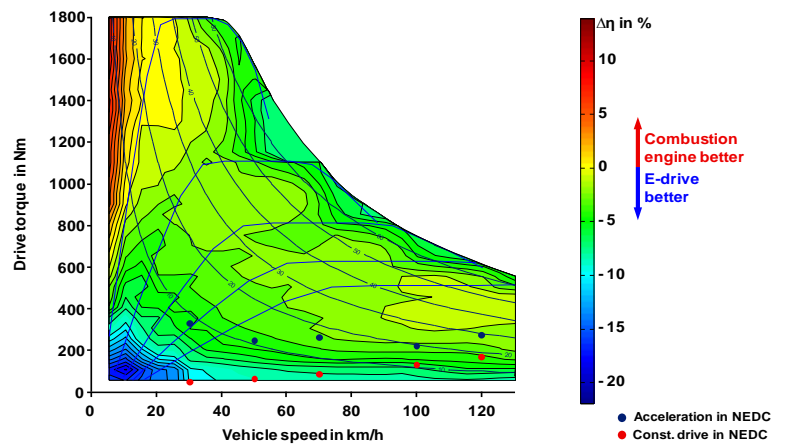

Fig.7 superposition map regarding efficiency 
The sizeable influence of electric power becomes apparent if the power provision efficiency for this parameter is changed. A power plant structure with an efficiency of $45 \%$ has been predicted for Germany for the year 2020, which would mean the advantages provided by electric vehicles compared with vehicles with internal combustion engines would increase to more than $7 \%$ in most operating points.

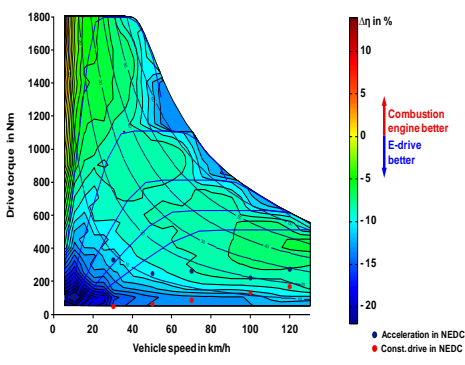

Fig.8a superposition power plant $45 \%$

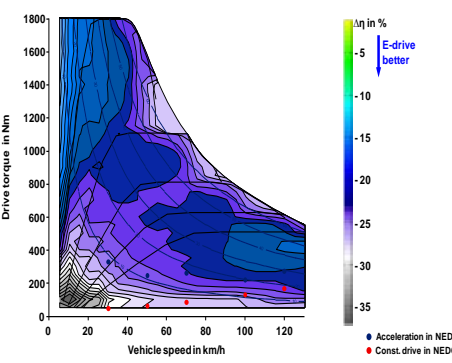

Fig.8b superposition power plant $60 \%$
An even clearer improvement is achieved if the efficiency of gas-fired power plants is used as a basis, which can reach an efficiency of up to $60 \%$. The advantage in terms of efficiency in this case would be around $17 \%$ in the differential data map. This observation not only demonstrates the relevance of the electric vehicle, the drivetrain or the batteries, but also the original parameter - the "provision efficiency".

\subsection{Comparison of $\mathrm{CO}_{2}$ emissions of internal combustion} engines and electric vehicles

The same investigation can be carried out for the target value of $\mathrm{CO}_{2}$ in today's power plant structure. Figure 9 shows the relevant differential data map of the drivetrain structures from Figure 5. A value of $590 \mathrm{gCO}_{2} / \mathrm{kWh}$ was used as a basis for the production of the electrical energy (based on the power plant structure in Germany in 2008).
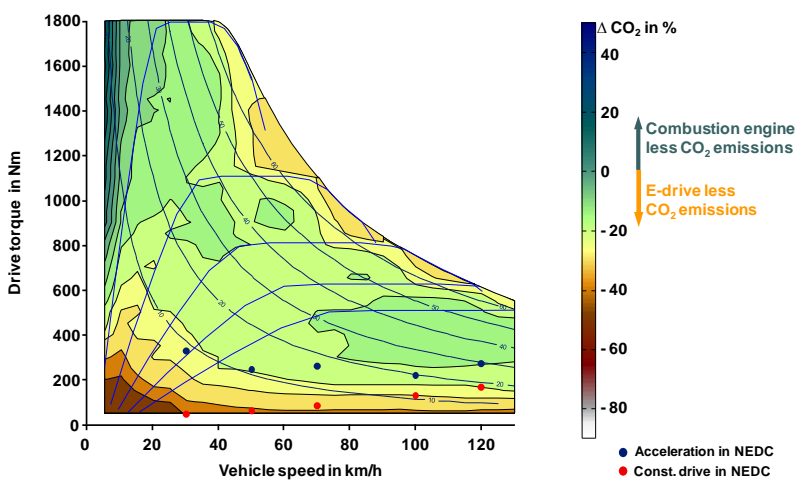

Fig.9 superposition map regarding CO2 [590 g CO2/kWh]
This already shows the advantage of an electric vehicle in the entire data map and, at $20-40 \%$ in the relevant areas of the NEDC, it is significant.

\subsection{Comparison of efficiency of electric drives}

The investigation of this data map can also be based on the definition of the structure of electric drivetrains. A comparison was made between the efficiency data map for an electric motor with a single-speed gearbox with the relevant equivalent with a 2-speed gearbox.
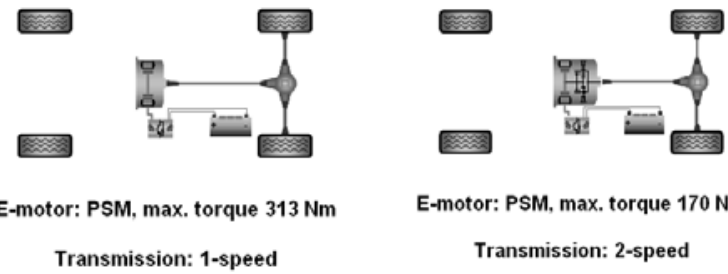

E-motor: PSM, max. torque $170 \mathrm{Nm}$ Transmission: 2-speed

Fig.10 comparative drivetrain structures 1-speed/2-speed

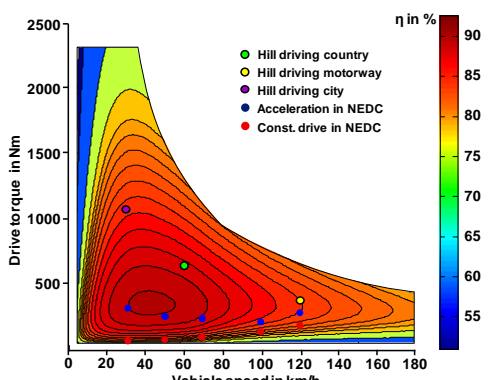

Fig.11 data map regarding efficiency 1-speed drivetrain

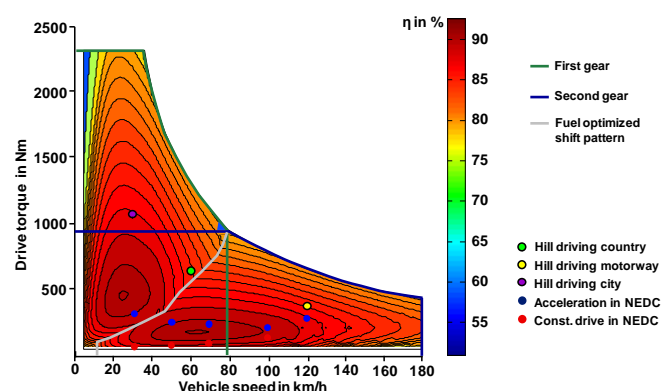

Fig.12 data map regarding efficiency 2-speed drivetrain

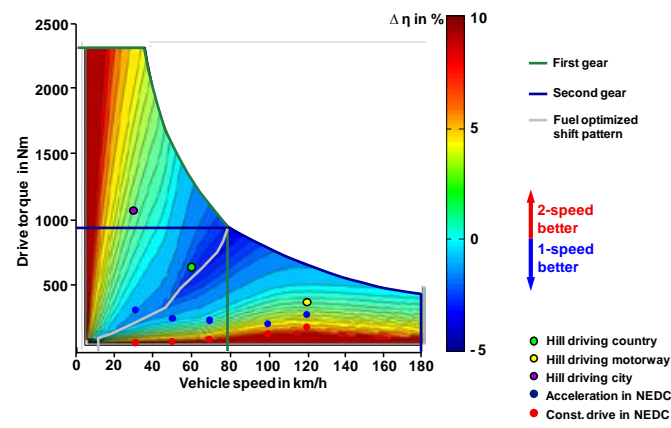

Fig.13 comparative drivetrain structures 1-speed/2-speed 
This comparison demonstrates an improvement to the efficiency in the NEDC of around 6\% when a 2-speed gearbox is used. Using the 2-speed gearbox also simplifies the dimensioning of the electric motor and the power electronics due to the lower maximum power required and increases the range.

\section{ELECTRIC VEHICLES}

During the development phase of a new concept for a spur gear differential, an idea emerged very early on that involved arranging this very compact axial differential coaxially to an electric motor. Even the first designs showed that an extremely compact electric drive can be used as an axle drive in this way. In accordance with the observations in Section 3.3., this axle drive was fitted with a 2-speed gearbox.

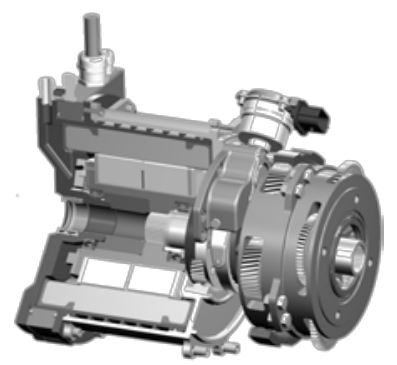

Fig.14 Coaxial electric drive with 2-speed gearbox and lightweight differential

In addition to using it as a purely electric drive, this drive can also be used as a hybrid element, for example, "through the road" in conjunction with a conventional drivetrain (e.g. automated manual transmission). A similar hybrid structure has recently been introduced into volume production as a diesel plug-in in the Peugeot 3008.

In addition to various hybrid concepts, we have also developed an all-electric vehicle with a permanently-excited electric motor as a test system.

Furthermore, the functions were expanded using modules to include torque vectoring. This is carried out relatively easily with a superimposing transmission (differential) that is controlled by a further electric motor.

The system enables the torque in transverse direction to be distributed in an energy-efficient manner thereby actively intervening in the vehicle dynamics and these functions have also been presented in relevant test systems.

The all-electric test vehicle was developed on the basis of an all-wheel drive with two active electric differentials, in order to demonstrate the possibilities and limits of electric torque vectoring on the front and rear axles and while operating on both axles simultaneously $^{(2)}$.
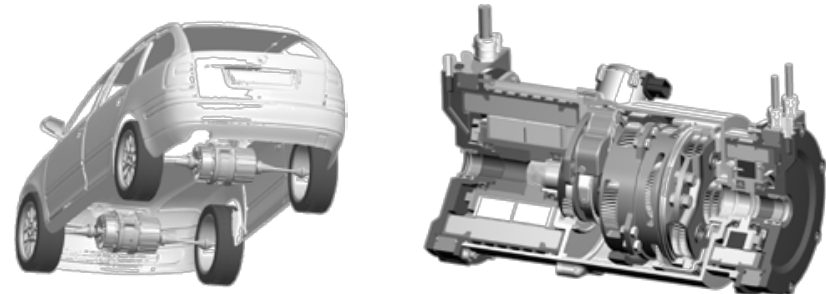

Fig.15 Test vehicle with electrically front and rear wheel drive ${ }^{(2)}$

A Skoda Octavia Scout 1.8 TSI AWD was selected as the platform for the test vehicle.

The all-wheel drive enables maximum freedom in testing the active distribution of torque on the front and rear axles. This means the vehicle can be tested and compared in front-wheel, rear-wheel and all-wheel drive modes. In addition, it enables a comparison to be made with a conventional drive without a superimposing transmission.

The active differential (1) in Figure 16 was fitted on both the front and rear axles.

The air-cooled lithium-ion battery (3) with a total capacity of $17.8 \mathrm{kWh}$ is located in the space usually taken up by the fuel tank and/or the vehicle tunnel. The battery comprises 110 cells with $3.6 \mathrm{~V}$ and $45 \mathrm{Ah}$ and therefore supplies a system voltage of around $400 \mathrm{~V}$. The technical data for the electric vehicle is summarized in Table 2.

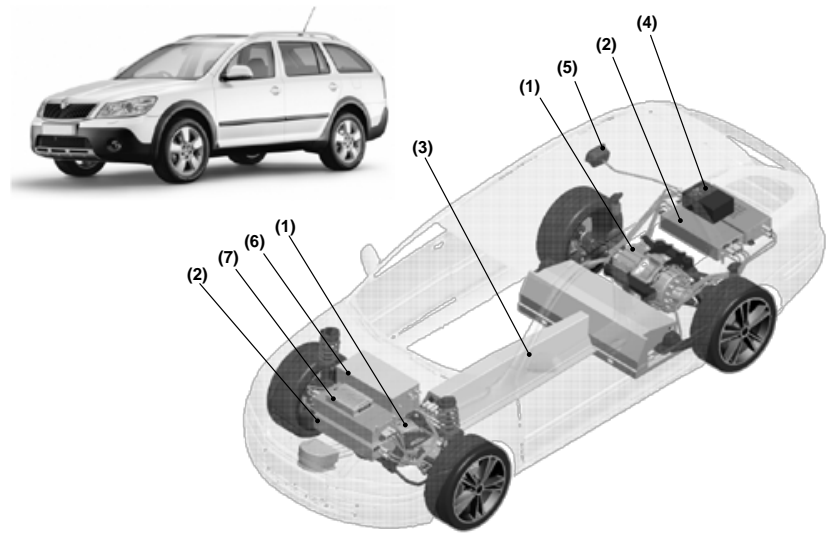

Fig.16 Electric vehicle with two active electric differentials with torque vectoring function on the front and rear axles: 1) Active electric differential, 2) converters, 3) battery, 4) battery charger, 5) socket for plug-in, 6) DC/DC converter, 7) ProTronic vehicle control unit ${ }^{(2)}$ 


\begin{tabular}{|l|l|l|}
\hline \multicolumn{3}{|c|}{ Specification of electric vehicle } \\
\hline Parameters & Description & Comments \\
\hline Vehicle & Skoda Octavia Scout & $\begin{array}{l}\text { orig. } \\
1,8 \text { TSI AWD }\end{array}$ \\
\hline Drive system & 2 active e-differentials & \\
\hline Output & $\begin{array}{l}\text { max. } 2 \times 110 \mathrm{~kW}(10 \mathrm{~s}) / \\
\text { nom. } 2 \times 140 \mathrm{~kW}\end{array}$ & \\
\hline Torque & $\begin{array}{l}\text { max. } 2 \times 170 \mathrm{Nm}(10 \mathrm{~s}) / \\
\text { nom. } 2 \times 100 \mathrm{Nm}\end{array}$ \\
\hline Curb weight & $1.900 \mathrm{~kg}$ & without a driver \\
\hline Speed & max. $150 \mathrm{~km} / \mathrm{h}$ & \\
\hline $\begin{array}{l}\text { Acceleration } \\
0 \text { to } 100 \mathrm{~km} / \mathrm{h}\end{array}$ & $8 \mathrm{~s}$ & \\
\hline
\end{tabular}

Fig.17 Technical data of the electric vehicle

The drive tests conducted were carried out both on a rolling test stand and on test tracks with the following results:

- The agility and driving comfort of the electric vehicle with deactivated torque vectoring is comparable with that of the original vehicle, despite the increase of in curb weight of around $350 \mathrm{~kg}$. The acceleration even increased by around 10\%.

- The accelerated rotor mass of the actuators and the resulting increased locking effect did not affect the driving characteristics and no noise was detected when torque vectoring was deactivated.

- The function of the novel electric torque vectoring on the front and rear axles was verified. The differences in torque were initially mapped in a simple data map as a function of the steering angle and vehicle speed. An improved torque vectoring strategy is currently being tested.

- The active differential can achieve differences in torque in the transverse direction of up to $2000 \mathrm{Nm}$, whereby the meaningful physical limit is around $1500 \mathrm{Nm}$.

- Torque vectoring on the rear axle stabilizes the vehicle and therefore improves vehicle safety. Torque vectoring on the front axle, on the other hand, supports the steering and contributes to vehicle agility and driving comfort.

The principle shown here with a coaxial electric motor and active electric differential is a drive concept that could form the optimum platform for future control strategies. The system combines the axle drive with the potential of intelligent transverse distribution of the torque. When used on both axles it also enables additional distribution of the torque in the longitudinal direction of the vehicle.

In addition to the coaxial drive, a great deal of development work is also being carried out on wheel hub motors.

\section{CONCLUSION}

The engines and transmissions of internal combustion engine drive trains are consistently being optimized in order to exploit the remaining potential for improving fuel consumption.

A systematic comparison of internal combustion engines and electric drives shows that the electric drive is already the far superior type of drive in terms of $\mathrm{CO}_{2}$ emissions. The results of the comparison for the efficiency are not as clear, however, developments in power plant technology show that the electric drive will be the superior alternative in other operating ranges in future $^{(3)}$.

Therefore, in view of the increasing public debate about the environment and energy resources, we can expect the proportion of hybrids with plug-in technology to increase considerably in the future. The only real obstacles that prevent these drive concepts from quickly gaining popularity are the insufficient power density and the excessive costs of the batteries.

However, current cost predictions and developments for "open" battery systems demonstrate that this is set to change by $2020^{(4)}$.

The our hybrid vehicle and the electric vehicle were built as development platforms for new drive concepts. The transmission and electric motor technology for the mobility of the future is currently being developed at Schaeffler on the basis of such complete systems.

This paper is written based on a proceeding presented at JSAE 2012 Annual Congress (Spring).

\section{REFERENCES}

(1)Wagner, Uwe; Reitz, Dierk : One Idea Many Applications Further development of the Schaeffler hybrid module, proceedings of Schaeffler Symposium, p426-p.439 (2010)

(2)Smetana, Tomas : Electric Drive with integrated electricmechanical Torque Vectoring. CTI Tagung (2011)

(3)FG I 2.5 : Strommix 2007. Umweltbundesamt(2007)

(4) McKinsey \& Company : Road towards a lowcarbon future :Reducing $\mathrm{CO} 2$ emissions from passenger vehicles in the globalroad transportation system. (2009) 